\title{
Mu-opioid receptor single nucleotide polymorphisms in the third intracellular loop affect receptor signalling in a pathway specific manner
}

\author{
Marina Santiago, Alisa Knapman, Mark Connor \\ Biomedical Sciences, Macquarie University, Australia
}

Introduction. Opioid analgesics are widely used in the clinic and interpersonal variation on therapeutic response have been observed. Single nucleotide polymorphism (SNP) of their molecular target, the mu-opioid receptor (MOPr), may be linked to this variation. In this study we further investigated two rare SNP of MOPr, located in the third intracellular loop (R260H and R265H), previously reported to affect receptor signalling (Knapman et al 2015).

Aims. Determine the effect of the R260H and R265H on MOPr signalling and regulatory pathways induced by opioid analgesics and endogenous opioids in vitro.

Methods. AtT20-FlpIn cells and CHOKI FlpIn T-Rex cells were stably transfected with human wild-type (WT), R260H and R265H MOPr. Radioligand binding assays ([3H]-DAMGO) were performed to determine receptor levels. A membrane potential-sensitive dye (FLIPR, molecular devices) was used to measure MOPr-induced K channel (GIRK) activation and adenylyl cyclase (AC) inhibition. ERK1/2 phosphorylation and loss of cell surface receptor were measured by whole-cell ELISA, and agonist-induced MOPr phosphorylation (S377) by Western Blot.

Results. The Bmax for [3H]-DAMGO binding was within 30\% of WT for each variant. Both variants showed a decrease in potency and maximum response for morphine activation of GIRK (Figure). Internalisation of R260H in response to DAMGO (30 min) was significantly reduced compared to WT (WT:36+/-8\%, R260H:14+/-8\%), and was associated with a decrease of $63+/-2 \%$ in S377 phosphorylation. Oxycodone stimulation of ERK1/2 phosphorylation was significantly $(\mathrm{P}<0.05)$ reduced at both SNPs (WT:101+/-10\%, R260H:43+/-4\%, R265H:65+/-5\%). Buprenorphine induced ERK1/2 phosphorylation was completely abolished at the $\mathrm{R} 260 \mathrm{H}$ variant, which showed the largest changes in signalling across all pathways.

Discussion. The importance of the ICL3 MOPr for G protein signalling was confirmed by this study. Amino acid changes in the N-terminal region of the ICL3 alter the signalling profile of MOPr in vitro, in a ligand-selective and/or pathwayspecific manner. R260H largely affects G-protein independent pathways. These SNPs have the potential to affect opioid response in patients but the effect in heterozygous genotype needs further investigation.

Knapman A et al (2015) Br J Pharmacol 172:349-363 